\title{
Introduction
}

\section{Oxidative Stress in Aging and Neurodegenerative Diseases: From Biology to Therapy, Perugia, Italy, May 2003}

Alzheimer's disease, like heart disease and cancer, is increasingly being shown as a lifestyle disease, related to genes but manifesting in a permissive environment. This issue presents some of our earliest understanding of the mechanisms responsible for the nexus of aging and Alzheimer's disease. Refinement of this theory beyond that of simple oxidative damage, oxidative stress, or increased antioxidant intact to an appreciation of cellular homeostasis in aging offers the hope of major inroads to our understanding and treatment of not only Alzheimer's disease, but a myriad of age-related chronic diseases.

George Perry and Mark A. Smith Case Western Reserve University Cleveland, Ohio, USA 